\title{
A broadband laser plasma x-ray source for application in ultrafast chemical structure dynamics
}

Wilfred Fullagar, Michael Harbst, Sophie Canton, Jens Uhlig, Monika Walczak, Claes-Göran Wahlström, and Villy Sundström

Citation: Review of Scientific Instruments 78, 115105 (2007); doi: 10.1063/1.2813340

View online: http://dx.doi.org/10.1063/1.2813340

View Table of Contents: http://aip.scitation.org/toc/rsi/78/11

Published by the American Institute of Physics

\section{Articles you may be interested in}

Laser plasma $x$-ray source for ultrafast time-resolved $x$-ray absorption spectroscopy

Structural Dynamics 2, 024301 (2015); 10.1063/1.4913585

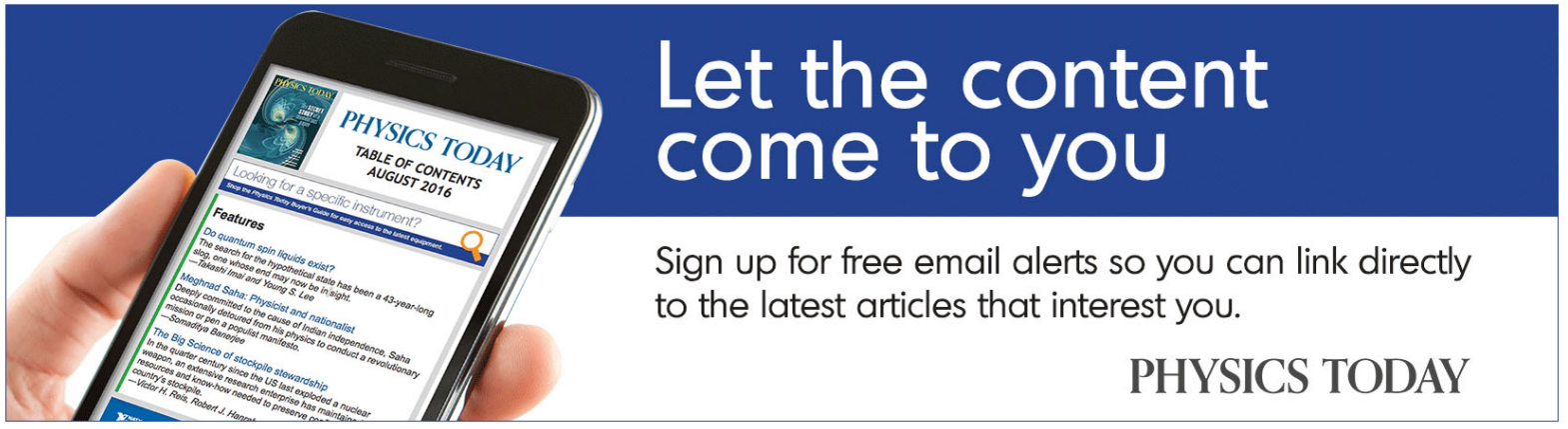




\title{
A broadband laser plasma x-ray source for application in ultrafast chemical structure dynamics
}

\author{
Wilfred Fullagar, Michael Harbst, Sophie Canton, Jens Uhlig, Monika Walczak, \\ Claes-Göran Wahlström, and Villy Sundström \\ Division of Chemical Physics, Lund University, P.O. Box 124, SE-22100 Lund, Sweden and Division \\ of Atomic Physics, Lund Institute of Technology, P.O. Box 118, SE-22100 Lund, Sweden
}

(Received 24 July 2007; accepted 16 October 2007; published online 20 November 2007)

\begin{abstract}
A plasma source free from characteristic emission lines is described, based on laser irradiation of a water jet in a helium atmosphere. Various key aspects of the laser interaction are presented along with practical characterization of the observed isotropic $\sim 4-10 \mathrm{keV}$ x-ray emissions, measurements of which indicate subpicosecond duration. Observations are consistent with a vacuum heating plasma mechanism at the helium-water interface and indicate strong potential for in-house ultrafast chemical structure dynamics application when coupled to contemporary detector developments. () 2007 American Institute of Physics. [DOI: 10.1063/1.2813340]
\end{abstract}

\section{INTRODUCTION}

At the present time, a strong motivation for the development of laser-based $\mathrm{x}$-ray sources is the possibility of inhouse measurement of molecular changes on picosecond and faster time scales, using $\mathrm{x}$-ray absorption spectroscopy ${ }^{1}$ and, where beam properties allow it, Laue crystallography. ${ }^{2}$ Both techniques require polychromatic radiation. In ultrafast experiments using monochromatic radiation (by angular dispersion of momentum transfer in powder ${ }^{3}$ or diffuse ${ }^{4}$ diffraction) there is an implicit need for collimation or focusing. ${ }^{5}$ Reflectometry also offers interesting possibilities. ${ }^{6}$ These experiments have in large measure only been implemented given a very high level of synchrotron development access, to date generally with pulse durations of $\sim 100 \mathrm{ps}$ that motivate continuing development of short pulse electron sources, time-slicing schemes, and streak cameras at large scale facilities. While $\mathrm{x}$-ray generation from condensed matter targets has been explored by many groups for several decades, ${ }^{7,8}$ much attention has recently been directed to the possibility of more or less collimated laser-generated broadband beams obtainable by relativistic electron acceleration, ${ }^{9-11}$ high harmonic generation, ${ }^{12,13}$ and reverse Compton scattering. ${ }^{14}$ The latter approaches are undergoing intensive development at the present time, and the possibility of in-house pump-probe Laue crystallography ${ }^{2}$ using the low shot rates but high collimated fluxes of broadband femtosecond betatron beams ${ }^{15}$ may be worth particular suggestion. Meanwhile the extreme laser power requirements, low flux soft x-ray beams, and need for implementation of a relativistic electron beam in these respective sources motivates ongoing work with simpler laser-based sources.

Temporal structure of plasma sources has in some instances been found to be much longer than hoped for ultrafast applications, ${ }^{16}$ likely because the emission of line radiation has the reacquisition of electrons by stripped atoms as a relatively slow prerequisite, ${ }^{17}$ this being dependent on plasma properties in the particular circumstances. The present work avoids production of detectable emission lines for reasons of source temporal duration, characterization, and application by exploiting bremsstrahlung from a water jet target. ${ }^{18-20}$ The choice of a low- $Z$ target for bremsstrahlung production is generally counterintuitive, ${ }^{21}$ however, we note that the total bremsstrahlung radiation yield from a fast electron brought to rest in water is only about an order of magnitude less than from the same electron brought to rest in denser, higher $Z$ materials such as mercury. ${ }^{22}$ The electron stopping length is greater in water (with potential consequences for pulse duration that we address later), but the escape depth of $\mathrm{x}$ rays is also greater. ${ }^{23}$ Such matters aside, the nontoxicity, high solvent power, ease of supply and handling, extremely smooth target surface, opportunities for debris-free continuous operation, and relatively relaxed safety considerations owing to the lack of intense hard emission line radiation make water a somewhat overlooked but nevertheless extremely attractive target material. ${ }^{24}$ In practice $\mathrm{x}$ rays have been observed from laser plasma targets in air $^{18,19}$ or preferably helium ${ }^{25}$ atmospheres, and $\mathrm{x}$ rays above $\sim 1 \mathrm{keV}$ have excellent transmission through the latter gas. The use of helium provides the opportunity to avoid vacuum systems and the corresponding remote control infrastructure in experimental setups, as well as permitting the study of vacuum incompatible samples. These features are all enjoyed in the apparatus described below.

Regarding detection, array detection methods not based on diffraction are vastly more efficient than single crystal diffraction-based energy dispersion, since the latter operate by discarding all photons in energy/angular phase space that do not meet the Bragg condition. Extended x-ray absorption fine structure (EXAFS) measurements are envisaged as an initial approach to combine such an array detector with a kilohertz repetition rate version of this source, in which its inherent beam divergence would be used to advantage. In such an arrangement, the extremely efficient detection would otherwise lead to detector saturation by "worthless" emission lines. For this and the various other reasons already given, 


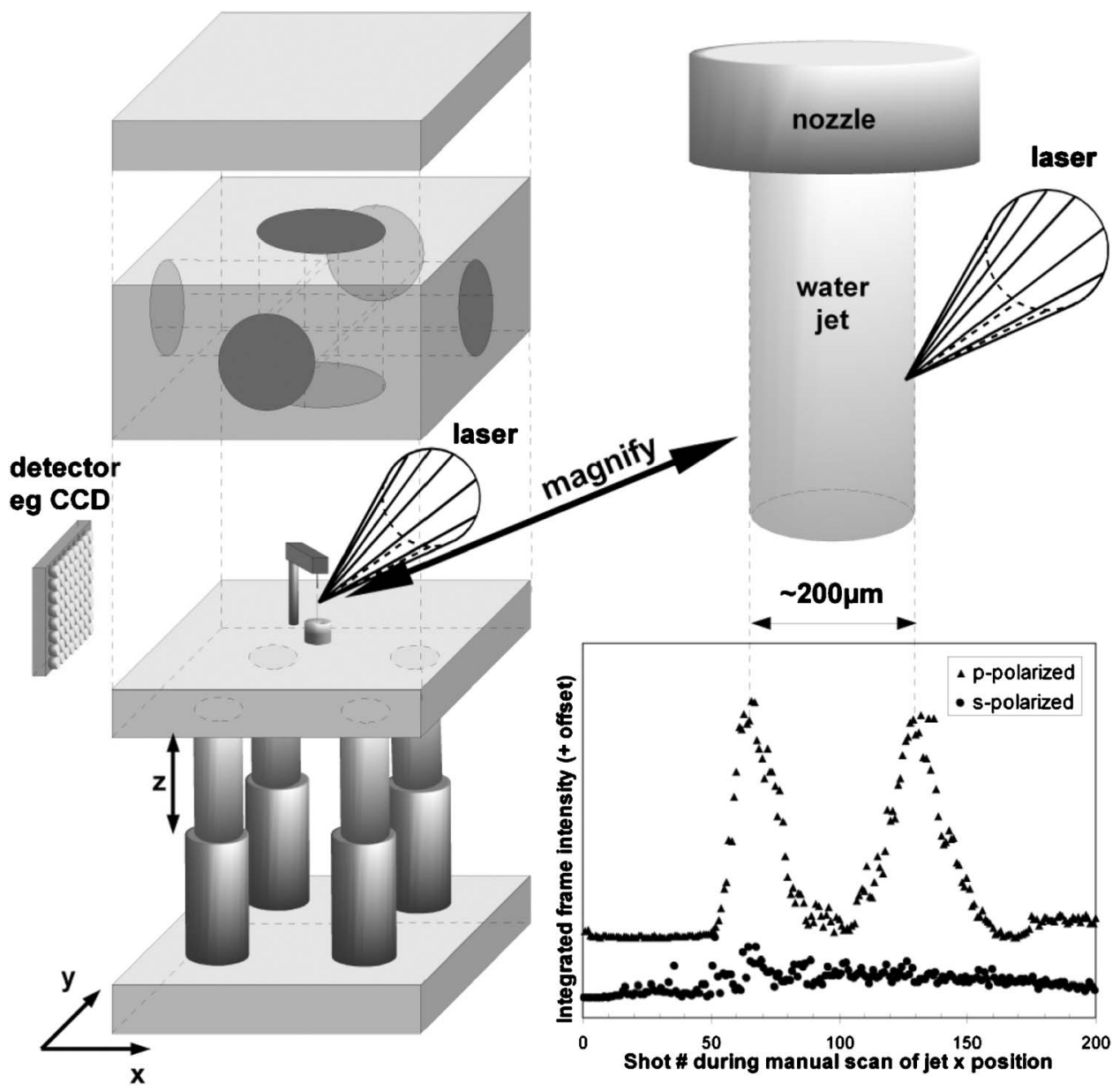

FIG. 1. Schematic of the laser plasma source. A horizontally polarized laser beam is focused to the tangent of a vertical water jet under helium in grazing incidence, resulting in isotropic $\mathrm{x}$-ray bremsstrahlung emission. The lead block surrounding the water jet has dimensions of $48 \mathrm{~mm} \times 96 \mathrm{~mm}$ $\times 96 \mathrm{~mm}$. Variants of this block permit evacuability, access of laser beams from additional angles, and placement of other items very close to the point of $\mathrm{x}$-ray emission.

and with the relevant detection developments underway, the source we describe deliberately avoids the generation of monochromatic hard $\mathrm{x}$ rays.

\section{APPARATUS}

Essential requirements for the $\mathrm{x}$-ray generator were adequate radiation shielding, close access to the source, rapid dismantling and reconstruction, independent movement of target and focusing optics, and no electronic remote control. Target chambers for different experiments were made by machining lead bricks, a typical example of which is illustrated in Fig. 1. Common features are a small hollow central region (typically $\sim 125 \mathrm{~cm}^{3}$ ) for the target, various holes to allow laser entry and $\mathrm{x}$-ray exit through suitable windows, provisions for accommodation of a small styrofoam diffuser block in the diverging laser beam, and admission and drainage of the target material through a lead base plate. The latter feature allows immediate access to the target jet by lifting away the upper part of the structure. The base plate is mounted on an $x y$-translation table on an optical table. Most variants have had deliberate small leaks, for example, where the water drains out of the chamber. This allows flooding of the internal cavity with helium by slight overpressure using a few $1 / \mathrm{m}$. Alternatively, by aspirating the water out of the drainage port using an aspirator pump, the jet can be pressurized by atmospheric pressure and operated close to water's vapor pressure at room temperature ( $\sim 25$ torr). This increases x-ray fluxes and temperatures very substantially, but requires further provisions to ensure jet stability; as such we choose to focus on the helium experiments here. Typical external linear dimensions of the lead constructions are 100-120 mm. One variant allows a closest external approach of $\sim 15 \mathrm{~mm}$.

Ordinary tap water is used in the jet, since the concentration of contaminants is negligible in the context of x-ray generation. A jet diameter of $150-200 \mu \mathrm{m}$ is usually used and can be either gravity fed or pumped. The laser is directed onto the jet 2-3 $\mathrm{mm}$ downstream from the nozzle, before it breaks into drops. Thicker jets increasingly lead to splattering. Thinner jets have not been tried by us, in the interests of maintaining good control of the laser interaction geometry. Occasional difficulties of internal condensation buildup on optical or x-ray windows are simply addressed by directing the incoming helium onto the offending optic.

The $10 \mathrm{~Hz} 800 \mathrm{~nm}$ Ti:sapphire laser of the Lund Laser Centre is used to generate the $\mathrm{x}$ rays. Light pulses traverse the following sequence of components: oscillator, acoustooptical modulator, (saturable absorber preamplifier), stretcher, regenerative amplifier, $(2 \times)$ cleanup Pockels cells, multipass amplifier, beam expansion telescope, compressor, variable polarizing beamsplitter. The saturable absorber preamplifier can be used to substantially reduce amplified spontaneous emission (ASE) from the oscillator and may be flipped in and out of the circuit at will; in addition a beam position stabilization circuit was installed during this work at the input to the butterfly amplifier. A specific consequence of 
these ongoing developments is that the present studies have enjoyed unusually low levels of prepulses and ASE pedestals $\left(<10^{-8}\right.$ contrast ratio), both of which critically affect $\mathrm{x}$-ray production in laser plasma sources. ${ }^{26,27}$ Key matters related to these aspects of the temporal quality of the laser beam are the temporal structure of the $\mathrm{x}$-ray generation, optimal target interaction geometry, and the production of energetic electron beams. Associated maximum energies available to our experiments have varied from $\sim 40$ to $\sim 300 \mathrm{~mJ} / \mathrm{pulse}$, while shot to shot stability is obtained by gain depletion in the final multipass amplifier stage. In combination with higher order compensations the compressor permits variable pulse durations from $<40$ fs to $\sim 1$ ps with positive or negative chirp. The $\sim 3 \mathrm{~cm}$ diameter output beam is variably polarized by a half-wave plate before transmission through a vertical Brewster plate to give a horizontally polarized transmitted beam used in these experiments, the intensity of which is varied by rotating the half-wave plate. The final focusing optic is a $50.8 \mathrm{~mm}$ diameter $90^{\circ}$ off-axis parabolic metal mirror with effective focal length of $\sim 100 \mathrm{~mm}$. The essential interaction geometry is shown in Fig. 1.

Our primary x-ray detector is a direct-detection, thinned, back illuminated, peltier cooled charge coupled device (CCD) consisting of $512 \times 51224.8 \mu \mathrm{m}$ pixels (Princeton Instruments). In use, the CCD camera unit is positioned in air a few millimeters distant from the exit window of the $\mathrm{x}$-ray source, typically in the horizontal plane orthogonal to the converging laser beam. Incident $\mathrm{x}$ rays traverse a $250 \mu \mathrm{m} \mathrm{Be}$ window and a few millimeters of vacuum before impinging directly on the $\mathrm{CCD}$, the latter typically being $\sim 7 \mathrm{~cm}$ from the source $(\sim 0.032 \mathrm{sr})$. Background-subtracted exposures of less than $6 \mathrm{~s}$ ensure that exposure-independent (e.g., preamplifier) noise is the dominant source of noise in the resulting frames. Distances and filters can be arranged such that individual pixels are single-photon detectors with energy resolution of $\sim 150 \mathrm{eV}$. When operated in this way, a histogram of the pixel intensities allows low resolution x-ray energy spectra on a single shot basis, after allowance for filters, events that span multiple pixels, and the CCD's spectral redistribution function. ${ }^{28,29}$ The practical rapidity of these measurements has enabled an extensive survey of fluxes and temperatures versus laser pulse energies and chirp. On the other hand, the available resolution is too poor for EXAFS and shot rates must be reduced to match the full frame CCD readout time. Moreover, an adequate account of the necessary data interpretation is at odds with the present intention of source characterization, so we choose instead to present the relatively very limited results obtained using a germanium point detector (below). The spectral results obtained from the two detectors are nevertheless in excellent quantitative agreement. For now it suffices to indicate the general utility of the multidetector array approach but also caution that the integrated CCD response should generally not be considered a proportional indicator of single shot $\mathrm{x}$-ray flux, since the source temperature may also vary, while different photon energies have different propensities to shift to lower energies in the course of spectral redistribution. This said, the utility of direct-detection CCDs for work of this nature must not be underestimated. Full frame readout of single shot $\mathrm{x}$-ray spectra requires 3-4 s, so a synchronous vertical hardware binning scheme is generally used to monitor the general $\mathrm{x}$-ray production in real time at the $10 \mathrm{~Hz}$ laser repetition rate. Spectral information is lost in this process. The resulting one dimensional array may then be summed in software to give the integrated detector response for the corresponding laser shot. Scans of observable yield versus arbitrary experimental variables can thus be made in real time on a shot by shot basis, while one dimension of spatial resolution can be retained if needed.

To confirm spectral observations made initially using the CCD in individual shots, much slower photon counting measurements were made at selected points in laser parameter space using a liquid nitrogen cooled germanium detector (ORTEC Canberra GUL0105 with EG\&G ORTEC 4293 MCA card). Despite a poorer energy resolution than the CCD and only a single "pixel," this detector has the advantages of response to harder $\mathrm{x}$ rays (up to $\sim 200 \mathrm{keV}$ ) and much less spectral redistribution. It was carefully starved into singlephoton counting mode using a $300 \mu \mathrm{m}$ pinhole in a lead sheet taped in front of the detector, which was variably positioned $\sim 10-30 \mathrm{~cm}$ from the source, with known thicknesses of aluminium for additional attenuation. Background counts were eliminated using temporal coincidence gating $(20 \mu$ s gate at $10 \mathrm{~Hz})$ and pileup was then arranged to be $\sim 10 \%$ by adjusting distances and filtering to give $\sim 1 \mathrm{event} / \mathrm{s}$ from the $10 \mathrm{~Hz}$ source. A few hours of measurement was sufficient to obtain the spectra presented here.

An x-ray streak camera (Kentech) was used to obtain an upper bound of x-ray pulse durations. The experiments described here used a $\sim 10 \mathrm{~nm}$ Au photocathode spaced $3 \mathrm{~mm}$ from the extraction grid, allegedly giving $\sim 0.1 \%$ quantum efficiency at 3-4 keV with temporal resolution of $\sim 25 \mathrm{ps}$. The latter value is consistent with the $1 \mathrm{~mm}$ photocathode slit width and $\sim 11 \mathrm{ps} / \mathrm{mm}$ maximum sweep rate. A low jitter timing pulse derived from the oscillator but gated by entrapment of the optical pulse in the regenerative amplifier was used for sweep triggering. Swept photoelectrons impinged on a phosphor coupled by a fiber plate to a temporally gated image intensifier (FlameStar multichannel plate and CCD).

\section{EXPERIMENTAL}

To align the off-axis parabolic focusing mirror, the horizontally polarized laser beam is brought to a focus in air and the mirror adjusted to produce a spark at the minimum possible energy. The laser self-focuses to different extents in windows and different gases, so the focal point shifts somewhat after placing the lead target chamber over the jet and admitting helium. The jet is moved into the spark (audible) and its position adjusted in real time so as to optimize the vertically binned CCD signal. Radiation incident on the beryllium window of the CCD camera is filtered by $\sim 6 \mathrm{~cm}$ of helium gas at ambient temperature and pressure, a beryllium or thin Mylar exit port, and a few millimeters of air. Other detectors (e.g., scintillator/photomultiplier tube, photodiodes, ion chambers, etc.) also give useful real time signals. When selecting a signal to optimize it is essential to be aware of 
electron beams with associated secondary radiations that we observe under particular laser conditions, or the various possible sources of ions in the chamber. ${ }^{30-33}$

Under the described experimental conditions, grazing incidence in $p$ polarization is necessary for significant $\mathrm{x}$-ray production. Thus when scanning the jet through the focus of the horizontally polarized laser focus, two distinct positions give similar yield, corresponding to the laser grazing either side of the jet, as shown in Fig. 1. Very little x-ray production can be observed when striking the jet normally or at intermediate angles. To confirm the grazing geometry, a thicker jet $830( \pm 10) \mu \mathrm{m}$ (microscope observation, circular jet cross section) was used and required a micrometer translation of $840( \pm 30) \mu \mathrm{m}$ between the two x-ray maxima. The dependence of the $\mathrm{x}$-ray yield on laser polarization is also shown in Fig. 1; when the previously intense and obvious $\mathrm{x}$-ray signal is again sought using vertically polarized light ( $s$-polarized interaction), only very weak and erratic x-ray emission is observed. Indeed it is clear that the slow decay of weak emission after passing through the beam is largely due water vapor remaining from the previous shot, which can build up locally when the helium flow rate is relatively low, as it was for the illustrated measurements. This was confirmed by repeating a given scan in the opposite direction, also by increasing the flow rate of helium. The erratic emission by the vapor is independent of the laser polarization.

The observable $\mathrm{x}$-ray yield depends critically on laser pulse duration (chirp) for pulse durations from the shortest value up to a few hundred femtoseconds. The integrated x-ray intensity scans shown in Fig. 2 were obtained by manually scanning the distance between the gratings in the laser pulse compressor through the value corresponding to the shortest pulse. Such scans are very valuable for diagnostic purposes and are performed routinely. The full width half maximum of the optical pulse was measured independently using an autocorrelator and is shown in the same graph. From this it is apparent that the laser pulse duration corresponding to the edge of the x-ray yield plateau (for either positive or negative chirp) is $\sim 200 \mathrm{fs}$, or $\sim 80$ field cycles. Shorter pulses have a stronger tendency to self-focus, so that it was not possible to perform simple scans of this nature in which the position of the jet was constantly optimized. As a result, such scans were done after first optimizing the jet position at a particular grating separation. This said, the transform limited pulse invariably gives relatively very poor yield, while the x-ray yield typically shows maxima or plateaus on either side of the shortest pulse. Such behavior is evident in all scans where the jet position is not first optimized for very short pulses, while the appearance of distinct yield maxima versus plateaus depends at least in part on the chirp at which the jet position is optimized prior to making such scans. Other experiments we describe typically involve use of a chirp corresponding to the edge of the x-ray yield plateau, this giving best $\mathrm{x}$-ray yield without unnecessarily compromising the laser (and therefore potentially also x-ray) pulse duration.

Shot to shot $\mathrm{x}$-ray production is very stable, enabling real time scans of various parameters and negating pileup problems that would otherwise require more extensive

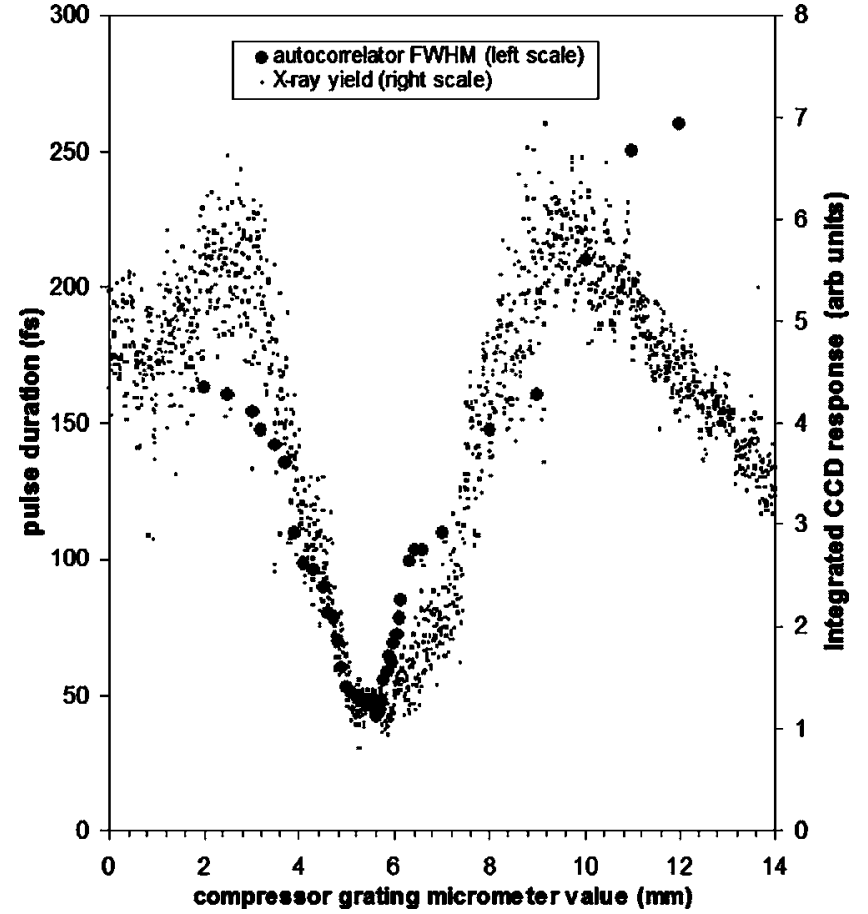

FIG. 2. Typical scan of $\mathrm{x}$-ray yield vs laser chirp, with overlaid measurement of laser pulse width using an optical autocorrelator. Indicated micrometer values are an offset from the full compressor grating separation of approximately $1 \mathrm{~m}$. For the x-ray yield data, the distance between the gratings was scanned manually while recording the integrated frame by frame CCD response in real time (see text). Bremsstrahlung yield is significantly reduced with optimally compressed laser pulses, an observation that remains true even after adjusting the jet position to compensate for self-focusing at the shorter pulse durations.

precautions ${ }^{34}$ in the multishot germanium point detector experiments described shortly. A root-mean-square stability of $5 \%-6 \%$ (shot-shot deviation of integrated CCD signal) is typical. This is believed to be due to a combination of shot noise for detection of x-ray events on the CCD with pulse to pulse stability of the laser energy.

When operated as described in this work, the source is essentially isotropic. This is evidenced in single shot CCD frames, multishot images, also films wrapped in aluminum foil lining the inside of the chamber. High-contrast small objects of known dimensions positioned close to the source cast shadows whose edge blurring allows geometrical estimates of the source size, being $\sim 30 \mu \mathrm{m}$ in both horizontal and vertical dimensions (CCD positioned orthogonal to the converging laser beam and jet direction). Analogous measurements, but with the camera repositioned in line with the converging laser beam, show that the depth of the x-ray radiating region in the water jet is $\sim 16 \mu \mathrm{m}$. To date experiments have not been performed to assess the source size on a single shot basis. Despite this, the alignment stability and vibration-free, laminar operation of the jet give confidence that the measured multishot source size is a close upper bound for the single shot size. We also observe that biological samples with thicknesses of hundreds of microns to millimeters can produce highly satisfactory images, since these "sample" depths are a fair match to the extinction lengths of the detected $\mathrm{x}$ rays in aqueous and organic material.

$\mathrm{X}$-ray energy spectra initially obtained using the direct- 
detection CCD as an array of energy dispersive detectors permitted extensive and rapid assessment of $\mathrm{x}$-ray flux and temperature within the laser pulse energy and chirp parameter space. These explorations show that integrated observable flux and spectral temperature both increase with energy per laser pulse at a fixed (optimized) laser chirp. Perhaps counterintuitively, flux and spectral temperatures also increase when the pulse duration is increased (chirped) away from the transform limited value at a fixed laser pulse energy. Such observations were without exception for pulse durations within the maximum $\mathrm{x}$-ray yield plateaus shown in Fig. 2, and for pulse energies up to the pulse energy available from the laser at the time of the CCD spectral measurements $(100 \mathrm{~mJ} / \mathrm{pulse})$. These observations were confirmed using the germanium point detector in measurements that permitted simultaneous use of both detectors. A CCD x-ray spectrum demands at most a few shots, while the corresponding germanium point detector spectrum requires several hours. Quantitative spectral and flux agreement of the two detectors is testament to both the long term stability of the source and adequate modeling of the CCD behavior. The long measurement times required by the point detector necessitate a very sparse and selective sampling of the laser's chirp and power parameter space. A selection of point detector spectra after compensation for filters, solid angle, and measurement time are shown in Fig. 3, where simultaneously measured data from the CCD are overlaid in one instance to illustrate the region of quantitative agreement and motivate relevant cautions in the spectral interpretation.

To date we have only seen evidence for a single exponential expression for the flux, modeled as $N=N_{0} \exp (-h \nu / k T)$, where $h, \nu$, and $k$ have their usual significance, and $N_{0}$ and $T$ are fitted flux and temperature parameters. Pileup manifests as a shallower slope at higher energies in the linear-log plot, being especially prominent in the illustrated CCD spectrum. A component with steeper slope can be apparent at the low energy end of spectra due to partially registered events and preamplifier noise in combination with ill conditioning (high filter normalization of low count rates). This is again most prominent in the CCD spectrum (in which events spanning multiple pixels were integrated using imaging software). The steeply declining ability of low energy photons to reach and stimulate the CCD (response function) is shown as partial motivation for this explanation, which finds further support in peripheral work with this detector using ${ }^{55} \mathrm{Fe}$ and ${ }^{241} \mathrm{Am}$ radioactive sources. When using titanium and copper foil filters of known thicknesses (results not shown), our ability to quantitatively model the magnitudes of the absorption edges is an assurance that other detectable radiations are not simultaneously present. Fitted values for the illustrated spectra are tabulated in Table I. Trends of temperature versus power and temperature versus chirp evident in the table verify the much more extensive CCD measurements; note the quantitative agreement of the corresponding CCD and germanium point detector spectra, measured at the same time. X-ray flux continues to be very appreciable even at the lowest pulse energy shown in the table, indicating that the results are transferable to high pulse repetition rate (kilohertz) laser systems.

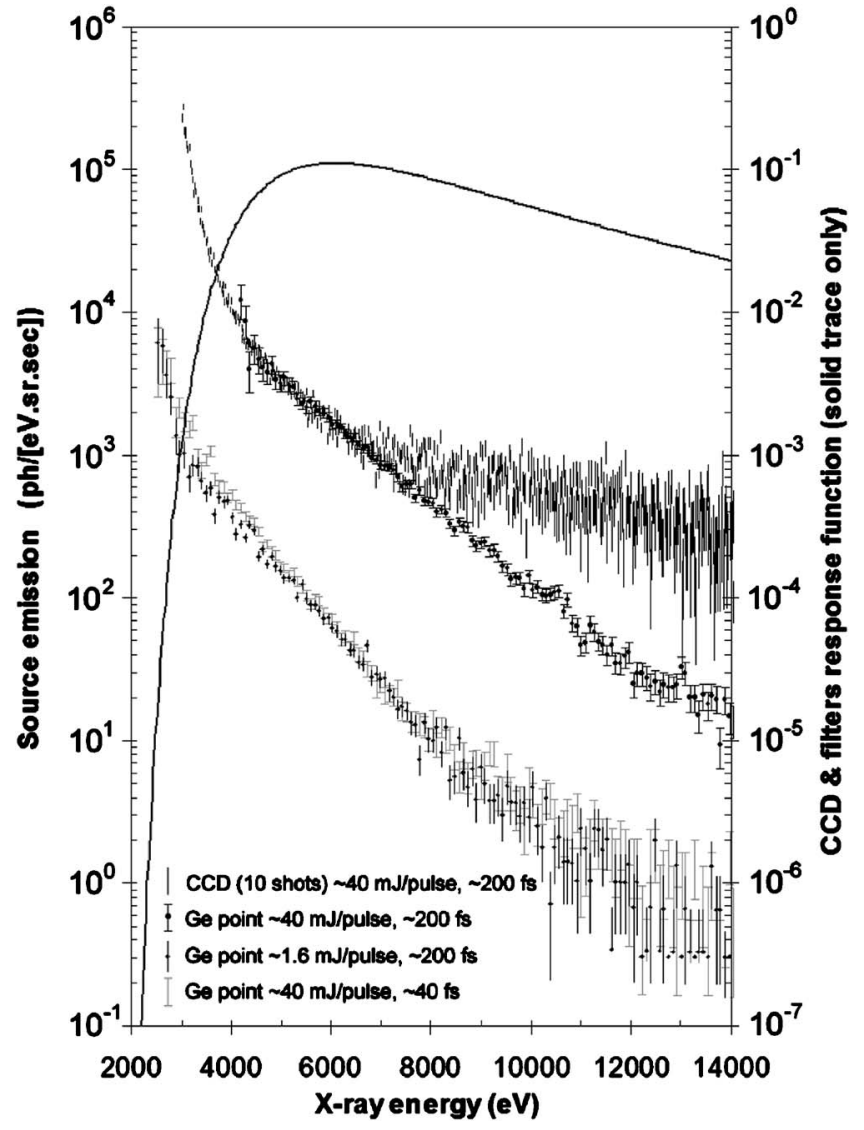

FIG. 3. Source spectra after normalization by filters, ADC binning interval, solid angle, and measurement time. A linear region is apparent in all cases from 4-7 keV and extends to higher energies depending on the degree of pileup. The response functions of the CCD and filters used in the associated spectrum are shown on a comparable scale. The data only support modeling of a single temperature component for each spectrum (see text).

An upper bound on the x-ray pulse duration was obtained using the streak camera. In practice it was possible to position the photocathode $\sim 12 \mathrm{~cm}$ from the plasma source. Optimization of the source permitted typically several tens of $\mathrm{x}$-ray events to be observed per laser shot. Following examination of the unswept photocathode image, the sweep was stepwise increased to its fastest value, with fine tuning of the delay at each step to temporally window the swept pulse. The image of the slit width did not significantly increase at any point during this procedure and the number of observed photons did not appreciably change. Significant timing jitter was observed on the fastest sweeps, as anticipated. This is shown in Fig. 4, where we also show an effective compilation following manual compensation of jitter in individual frames. As far as is possible to determine, the x-ray pulse obtained in this way is symmetrical, moreover narrower than

TABLE I. Fitted source flux and temperatures corresponding to Fig. 3.

\begin{tabular}{cccc}
\hline \hline $\begin{array}{c}\text { Energy/pulse } \\
(\mathrm{mJ})\end{array}$ & $\begin{array}{c}\text { Optical pulse duration } \\
(\mathrm{fs}, \text { estimate })\end{array}$ & $\begin{array}{c}\mathrm{N}_{0} \\
(\mathrm{ph} /[\mathrm{ev} \mathrm{sr} \mathrm{s}])\end{array}$ & $\begin{array}{c}T \\
(\mathrm{~K}[\mathrm{eV}])\end{array}$ \\
\hline 40 & $\sim 200$ (max x rays) & $17.5 \times 10^{3}$ & $17.4 \times 10^{6}[1500]$ \\
1.6 & $\sim 200$ (max x rays) & $14.7 \times 10^{3}$ & $12.7 \times 10^{6}[1090]$ \\
40 & $\sim 40$ (shortest pulse) & $29.2 \times 10^{3}$ & $11.5 \times 10^{6}[990]$ \\
\hline \hline
\end{tabular}



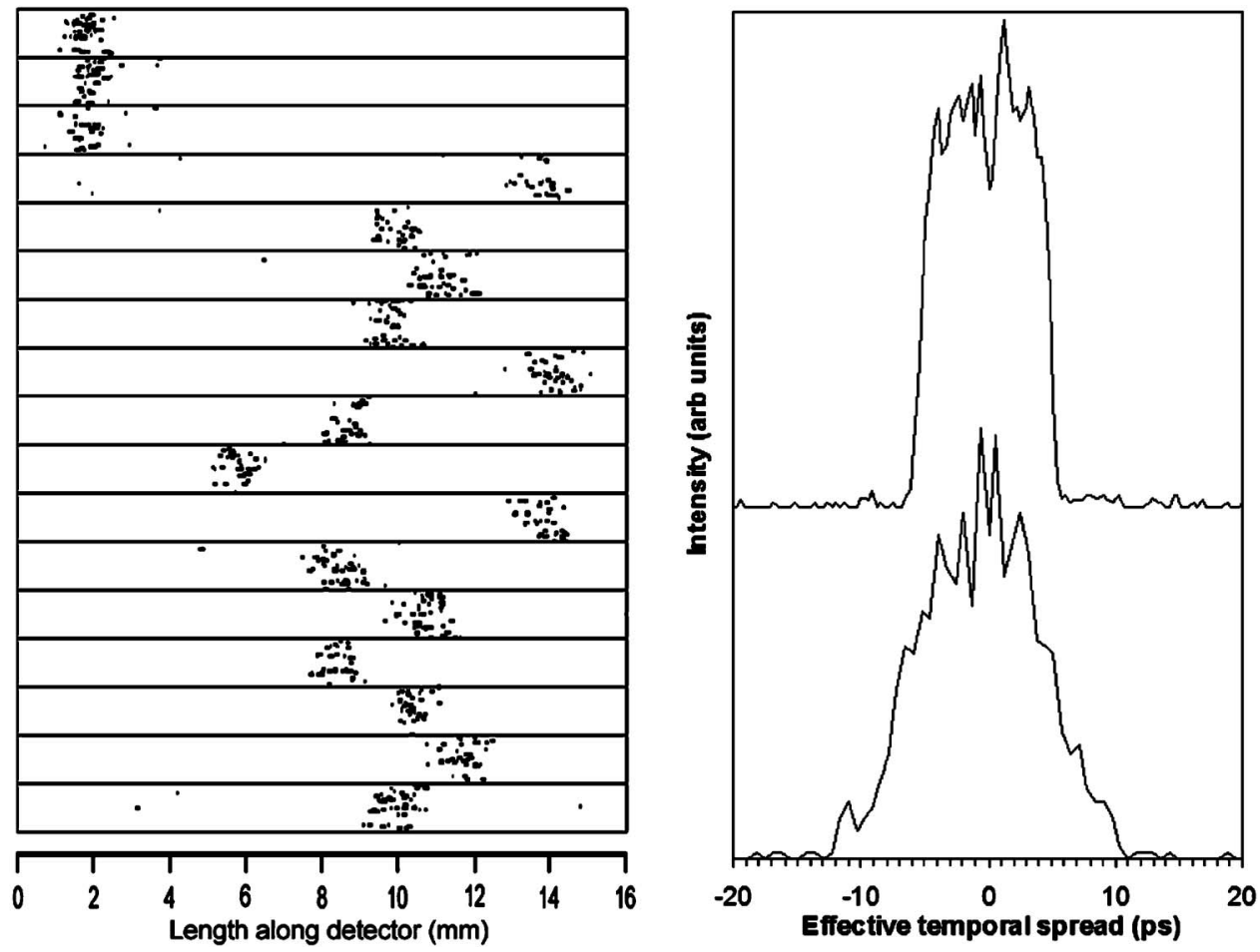

FIG. 4. Summary of streak camera results. Typically several tens of events were registered per shot. On the left are examples of unstreaked (top three) and streaked images at the fastest rate of $\sim 11 \mathrm{ps} / \mathrm{mm}$. Trigger jitter causes differences in the positions of the swept photocathode images at this sweep rate. The upper right trace shows the breadth of the unswept photocathode image, while the lower trace shows apparent broadening that could be inferred by subjective manipulation of 22 swept images.

the $\sim 25$ ps resolution function previously ascribed to this instrument. Various instrumental matters can contribute to such spreading in the streaked images, but on the other hand the observation of only a few photons per shot could potentially reduce the width in the compilation. Determination of what limits the camera's X-ray temporal response function lies outside the goals of the present work. We believe these measurements indicate an upper bound for the x-ray pulse duration, whose actual duration is presumed to be considerably shorter, as discussed below.

To motivate ongoing developments leading to applications, Fig. 5 shows CCD shadow images obtained in several hundreds of shots, indicative of the practical flux and isotropicity, also a single shot absorption spectrum of a $5 \mu \mathrm{m}$ titanium foil measured using the CCD. The $K$-absorption edge at $4.97 \mathrm{keV}$ in the latter is clearly evident. To our knowledge, this is the first literature presentation of a single shot, presumed subpicosecond absorption edge measurement using an in-house vacuum-free laser plasma system.

\section{DISCUSSION}

Unusually low prepulse and ASE pedestal ratios in our laser system $\left(<10^{-8}\right)$ indicate a sharp plasma density gradient during laser-target interaction at the water surface. Such conditions are required for the vacuum heating mechanism of Brunel, ${ }^{35}$ which we invoke as the mechanism for electron acceleration preceding $\mathrm{x}$-ray bremsstrahlung generation. Strong evidence is found in the grazing angle of incidence and polarization requirements ${ }^{36}$ on the extremely smooth target surface, in x-ray measurements that are not complicated by the simultaneous observation of emission line radiation. Pulse durations corresponding to the edge of the plateau ( $\sim 200 \mathrm{fs}$ ) along with estimates of the laser spot size and measurement of laser power $(\sim 100 \mathrm{~mJ} /$ pulse $)$ indicate target
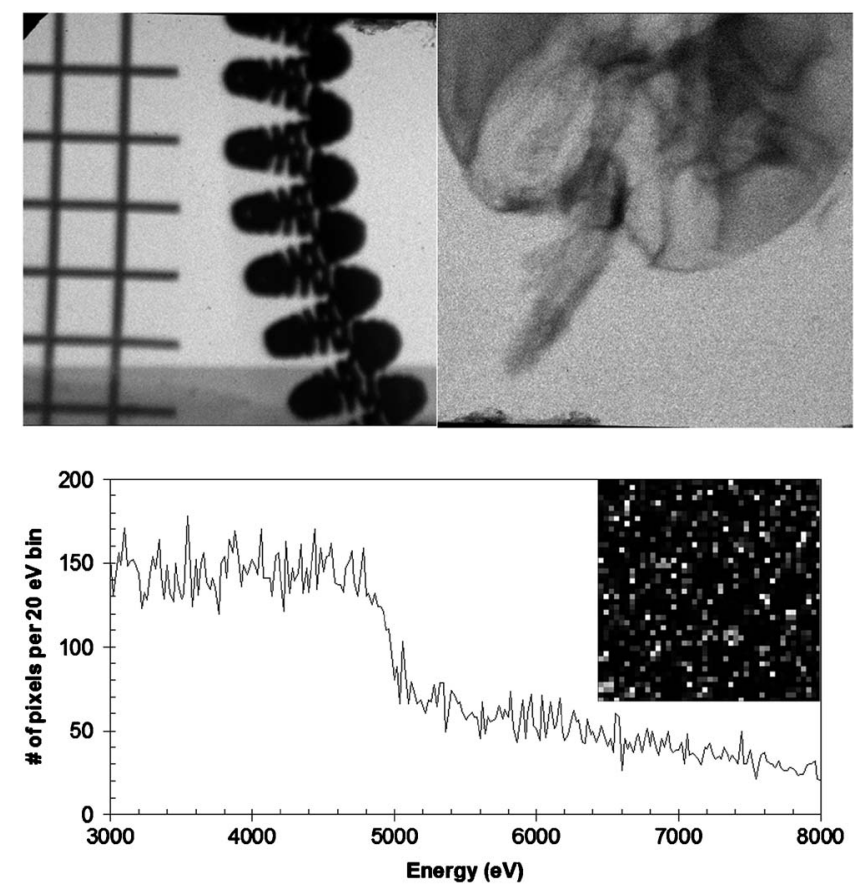

FIG. 5. Motivations for source applications. Shadow imaging applications demonstrate an abundance of isotropic $\mathrm{x}$ rays with a small source size. Upper left image shows a $254 \mathrm{~mm}$ nickel mesh a $60 \mathrm{~W}$ incandescent lamp filament, showing its supercoil structure, here measured in the horizontal plane orthogonal to the converging laser beam. A total exposure time of 2 min was used. The high contrast of such objects permits estimates of the source size in three dimensions. At upper right is the head of a housefly, for which a reasonable match of the extinction depths of observable $\mathrm{x}$ rays to sample dimensions permits good contrast of internal features. An aluminum filter $(12 \mu \mathrm{m})$ was used in this total $4 \mathrm{~min}$ exposure. The lower image is a $K$-edge absorption spectrum of a $5 \mu \mathrm{m}$ titanium foil obtained as a direct histogram of pixel intensities in a single, presumed subpicosecond burst (see text). The inset gives an impression of the fairly high degree of pileup in this measurement. 
intensities of $\sim 10^{17} \mathrm{~W} / \mathrm{cm}^{2}$ for our experiments, an intensity range in which this heating mechanism is well established. ${ }^{7}$ Also importantly, we observe x-ray temperatures that increase as the laser pulse duration is extended while maintaining constant laser pulse energy, an observation consistent with resonant heating of electrons in the laser field. The present combination of observations is thus a clear practical indication of this mechanism.

In addition to spectral properties, temporal aspects of the source are vital to its development, since longer pulse laboratory sources requiring less infrastructure can also be constructed. ${ }^{37,38}$ At the present time there are four independent handles on this question. The first is the streak camera measurements, which merely permit the statement that the observed x-ray pulse duration is less than or at most comparable to that instrument's temporal resolution function. Secondly, there is the duration of the optical pulse, whose envelope is observed to be 200-300 fs when x-ray generation is optimized. As shown in Fig. 2 this can be reduced while still obtaining appreciable x-ray production. Thirdly, we infer electron temperatures from the $\mathrm{x}$-ray spectra (typically $\sim 1 \mathrm{keV}$ ). While acknowledging the potential for misinterpretation, this suggests a fast rate of blackbody radiation loss through the Stefan-Boltzmann law in combination with the measured source size. The energy of a $100 \mathrm{~mJ}$ laser pulse would then be dissipated in $<10 \mathrm{fs}$, assuming complete conversions. Fourthly, the electron attenuation length in water, divided by the velocity of electrons capable of producing high energy x rays, indicates the time scale of the interaction. The continuous slowing down approximation electron penetration distances ${ }^{22}$ suggest approximate time scales of 42 , 330 , and 760 fs for the bremsstrahlung interactions of 10, 50, and $100 \mathrm{keV}$ electrons in a $200 \mu \mathrm{m}$ water jet. In the same vein, our multishot $\mathrm{x}$-ray radiation depth estimate of $\sim 16 \mu \mathrm{m}$ associated with registry of mostly $4-5 \mathrm{keV} \mathrm{x}$ rays suggest a time scale of $\sim 400 \mathrm{fs}$. From such arguments it would appear that either the electron stopping depth or perhaps the optical pulse duration determines the x-ray pulse duration. Since water jets can be arranged that are thinner than the electron stopping length, while buildup of electron energies requires several field cycles, the latter bounds are subject to practical manipulation and further interpretation, respectively. Access to an X-ray streak camera with subpicosecond temporal resolution or careful single shot radiation depth measurements could perhaps give a practical answer to which bound is more appropriate in particular circumstances. In either case it appears that $\mathrm{x}$-ray pulses are around two orders of magnitude briefer than typically obtained in synchrotron storage rings, this being a range that renders them of particular interest for molecular chemical structuredynamics studies. As such, and with the relevant detection developments underway, it is presumed that applicationbased pump-probe experiments will provide a fifth and independent practical indication in due course. Synchronization of the x-ray pulse with the laser in the associated pumpprobe topologies will be essentially free from temporal jitter.

The number of broadband $\mathrm{x}$-ray photons emitted in an experimentally accessible solid angle is already quite adequate when considering essential numbers needed by appli- cations such as pump-probe transmission EXAFS. Detection schemes based on conventional diffraction topologies are the only restriction to this application. The essential truth of this assertion is quickly appreciated from Fig. 5, where we show images involving necessarily large numbers of photons obtained in very reasonable experimental times at $10 \mathrm{~Hz}$, along with an absorption edge observation simply obtained using a single laser shot. Associated work with our CCD detector confirms that the addition of many such histograms is of no practical value with this kind of detector. Nevertheless it is clear that one approach to ultrafast EXAFS on such a laser plasma based source is to subtend a sufficient solid angle around the source with a suitable number of high-resolution detectors that are capable of readout at the laser repetition rate, such that the dramatic losses associated with analyzer crystals are completely avoided. In such a setup the water jet itself might be a very inviting solvent matrix for certain EXAFS samples if $x$ rays are generated on the side of the jet such that they must traverse it to reach the detector; alternatively a second jet or other sample apparatus is easily arranged in the closest possible proximity to the source. In either case, the volume of sample requiring optical excitation needs to have dimensions only slightly larger than the $\sim 30 \mu \mathrm{m} \times 30 \mu \mathrm{m} x$-ray source, enabling high levels of optical sample excitation using available ultrafast lasers, while in principle requiring very little sample material and leading to very small temporal dispersion due to geometrical considerations. The lack of $\mathrm{x}$-ray focusing optics in this scheme (in particular, before the sample) eliminates issues related to alignment and temporal dispersion in such components. Our source is extremely well suited to such a detection scheme, since suppression of energies outside the range of interest involves nothing more than simple foil filters, removal of unwanted emission lines is unnecessary, and the isotropic nature of the x-ray emission would be used as an advantage. Given sufficient pixel readout rate, it is readily appreciated that the rate of data acquisition is proportional to both the number of pixels and the laser repetition rate. We have demonstrated that laser pulses do not need to be unusually brief or powerful, so that standard kilohertz repetition rate lasers can be used, if necessary with tight focusing and measures to improve the contrast ratio.

Direct-detection CCD detectors are presently giving way to much higher resolution microbolometric array detectors ${ }^{39}$ which will obviate the need for wasteful crystal diffractionbased analyzers hitherto associated with suitably resolved energy dispersion at "hard" x-ray energies. Foreseeable availability of microbolometric array detectors ${ }^{40}$ is a key motivation for ongoing exploration of this source. The millikelvin temperatures required by such detectors gives cause to reflect on the extraordinary temperature range these experiments would involve. In combination with in-house sources such as described here and by others, ${ }^{12}$ these detectors should enable ultrafast molecular structure studies in any suitably equipped ultrafast kilohertz laser laboratory. To assist the necessary developments, a few precautions of particular relevance to the source described here are outlined in the remaining paragraphs. We further point out that with the contemporary development of collimated broadband laser- 
generated x-ray beams, such array detectors will enable x-ray momentum transfer measurements, this being the essential measurement required for structural measurements by diffuse scattering, crystallography, and reflectometry. As such, the development of fast readout high-resolution array detectors in photon-starved ultrafast x-ray science should offer benefits comparable to the development of time of flight techniques in neutron science.

In a somewhat shorter term, conventional extremely lossy but technically straightforward diffraction-based technologies ${ }^{41,42}$ are lately enjoying a renaissance ${ }^{43,44}$ which might also permit practical ultrafast $\mathrm{x}$-ray absorption spectroscopy applications. The latter developments motivate awareness of source parameter spaces that can be used to enhance the bremsstrahlung, but also of the practical complications that may ensue. For example, we observe that hard $\mathrm{x}$-ray fluxes and temperatures increase considerably when using a water aspirator vacuum instead of helium, an approach in which it is necessary to degas and slightly cool the water in order to maintain jet stability. Also, when using diffraction-based detection the generation of hard emission line radiation is not in itself an issue (indeed such lines are useful energy calibrants). Thus higher- $Z$ materials or concentrated solutions of high- $Z$ compounds are typically attractive targets because of their higher bremsstrahlung yields. In practice, it proves difficult to avoid problems associated with debris.

The controlled insertion of a prepulse with $s$-polarized interaction geometry (capable of generating a preplasma without itself generating significant $x$ rays) introduces an enormous parameter space displaying very rich behavior. In a simplistic view, prepulses generate a more gradual local plasma density gradient that causes refraction of the subsequent pulse away from the higher density regions at greater depths. One consequence is that $\mathrm{x}$-ray generation by the subsequent $p$-polarized pulse is optimal at a nongrazing angle of incidence. Indeed we observe that the controlled introduction of prepulses is associated with dramatic changes in the interaction geometry leading to $\mathrm{x}$-ray generation. In this context we note that $30^{\circ}-60^{\circ}$ angles of incidence have often been cited in the literature, ${ }^{7,25,45}$ in clear contrast to the grazing incidence required by the present very low prepulse experiments and the sharp density profile vacuum heating mechanism invoked to explain our specifically bremsstrahlung results. In addition, upon the introduction of prepulses or when the contrast of our laser system is impaired, additional caution is needed to distinguish $\mathrm{x}$ rays generated at the target from collimated $\sim 20-200 \mathrm{keV}$ electron beams ${ }^{31,32,45}$ and secondary emissions these can give rise to, both of which we have unambiguously observed under those specific conditions. While indicating potential pitfalls for the unwary, this also indicates the feasibility of controlled electron beam generation desirable in related contemporary developments. As a result, such beams are the subject of peripheral investigations.

The approximate brilliance of the source when operating under helium is inferred from Table I and the observed multishot source size of $30 \mu \mathrm{m}$, which perhaps varies under different laser conditions. Thus the first entry in the table cor- responds to $\sim 397$ photons $/ \mathrm{s} \mathrm{mm}^{2} \mathrm{mrad}^{2} 0.1 \%$ bw at $5 \mathrm{keV}$. Such low values give an initial impression of woeful inadequacy compared with contemporary synchrotron and other emerging sources. However, the comparison is not a meaningful one. The reason is that such sources normally aim for increasingly tight collimation and narrow bandwidths for the purposes of satisfying grazing incidence mirror requirements and crystallographic Bragg conditions in analyzer optics, such that the corresponding normalizations dramatically favor them. As we indicate, diffraction based energy dispersion is not viewed as the best way to proceed with chemical structure studies using this source. Topologies that address large solid angles are clearly essential, along with low temporal dispersion before traversing samples, and we have indicated how both can be achieved. Fluxes from our source will scale linearly with laser repetition rate and can be made to increase by adjustment of several independent laser parameters as we have shown.

In closing we point out that earlier solid target plasma sources involved mechanisms to address target replacement, debris handling, remote control, and target and/or sample evaporation in evacuated chambers, as well as pumping equipment and necessarily more extensive radiation safety precautions. Given such infrastructure, typical shot to shot fluctuations from solid targets could still be several orders of magnitude. ${ }^{34}$ Many observations indicate substantial intensity increases when shooting into nanostructures, ${ }^{46}$ existing craters, ${ }^{47}$ and (very early in this work) lead smoke from laser ablation of the target chamber itself, collectively demonstrating the criticality of reliable interaction geometry in reproducible experiments. The source we describe has sought to address all these matters in simple and robust ways, this being a prerequisite for foreseeable applications in chemical structural dynamics. Substantial alterations and additions to the laser system as well as construction of several variants of the $\mathrm{x}$-ray generator during the two years of these developments confirm the robustness of the observations presented.

\section{CONCLUSIONS}

An x-ray laser plasma source is presented whose novelty lies in its broadband spectral qualities free from observable emission lines, practical simplicity with complete avoidance of vacuum systems, or remote control, temporal duration suitable for ultrafast applications, good shot to shot stability and transferability to lower power, higher repetition rate laser systems. To our knowledge this work is the first to demonstrate a clear requirement for grazing incidence of $p$-polarized laser fields for the generation of few keV bremsstrahlung in the absence of significant prepulses or ASE. This gives strong indications of a vacuum heating mechanism, which finds further support in quantitative measurements of yield and x-ray temperatures versus laser chirp. We indicate prepulse based approaches that may allow the source to be made brighter without sacrificing the associated x-ray temporal structure. A single shot observation of a Ti foil absorption edge is presented to stimulate foreseeable spectrometer and detector developments in anticipation of ultrafast structural dynamics applications. In association with contempo- 
rary detector developments, and along with other existing laser-based x-ray sources, we believe the source we describe points the way to in-house picosecond chemical structural dynamics in essentially any modern ultrafast laser laboratory operating at kilohertz laser repetition rates.

\section{ACKNOWLEDGMENTS}

We thank A. Persson, E. Pourtal, F. Lindau, O. Lundh, T. Hanson, and J. Larsson for assistance with laser operation, optical characterizations, discussions, and general developments during this project. This work has enjoyed financial support from the Knut and Alice Wallenberg Foundation, the Kungliga Fysiografiska Sällskapet, and the Swedish Research Council.

${ }^{1}$ C. Bressler and M. Chergui, Chem. Rev. (Washington, D.C.) 104, 1781 (2004).

${ }^{2}$ V. Srajer, Z. Ren, T. Y. Teng, M. Schmidt, T. Ursby, D. Bourgeois, C. Pradervand, W. Schildkamp, M. Wulff, and K. Moffat, Biochemistry 40, 13802 (2001).

${ }^{3}$ S. Techert, F. Schotte, and M. Wulff, Phys. Rev. Lett. 86, 2030 (2001).

${ }^{4}$ H. Ihee, M. Lorenc, T. K. Kim, Q. Y. Kong, M. Cammarata, J. H. Lee, S. Bratos, and M. Wulff, Science 309, 1223 (2005).

${ }^{5}$ A. Franks, Proc. Phys. Soc. London, Sect. B 68, 1054 (1955).

${ }^{6}$ R. F. Garrett, J. W. White, D. J. King, T. L. Dowling, and W. Fullagar, Nucl. Instrum. Methods Phys. Res. A 467, 998 (2001).

${ }^{7}$ P. Gibbon and E. Forster, Plasma Phys. Controlled Fusion 38, 769 (1996).

${ }^{8}$ S. C. Wilks and W. L. Kruer, IEEE J. Quantum Electron. 33, 1954 (1997).

${ }^{9}$ W. P. Leemans, E. Esarey, J. van Tilborg, P. A. Michel, C. B. Schroeder, C. Toth, C. G. R. Geddes, and B. A. Shadwick, IEEE Trans. Plasma Sci. 33, 8 (2005).

${ }^{10}$ A. Rousse, K. T. Phuoc, R. Shah, A. Pukhov, E. Lefebvre, V. Malka, S. Kiselev, F. Burgy, J. P. Rousseau, D. Umstadter, and D. Hulin, Phys. Rev. Lett. 93, 135005 (2004)

${ }^{11}$ W. P. Leemans, B. Nagler, A. J. Gonsalves, C. Toth, K. Nakamura, C. G. R. Geddes, E. Esarey, C. B. Schroeder, and S. M. Hooker, Nat. Phys. 2, 696 (2006).

${ }^{12}$ E. Seres, J. Seres, and C. Spielmann, Appl. Phys. Lett. 89, 181919 (2006).

${ }^{13}$ J. Seres, P. Wobrauschek, C. Streli, V. S. Yakovlev, E. Seres, F. Krausz, and C. Spielmann, New J. Phys. 8, 251 (2006).

${ }^{14}$ K. Chouffani, F. Harmon, D. Wells, J. Jones, and G. Lancaster, Laser Part. Beams 24, 411 (2006).

${ }^{15}$ K. T. Phuoc, F. Burgy, J. P. Rousseau, V. Malka, A. Rousse, R. Shah, D. Umstadter, A. Pukhov, and S. Kiselev, Phys. Plasmas 12, 023101 (2005).

${ }^{16}$ J. Larsson and A. Sjogren, Rev. Sci. Instrum. 70, 2253 (1999).

${ }^{17}$ A. K. Shuaibov, L. L. Shimon, A. I. Dashchenko, I. V. Shevera, and M. P. Chuchman, Plasma Phys. Rep. 27, 82 (2001).

${ }^{18}$ K. Hatanaka, T. Miura, and H. Fukumura, Appl. Phys. Lett. 80, 3925 (2002).

${ }^{19}$ K. Hatanaka, T. Miura, and H. Fukumura, Chem. Phys. 299, 265 (2004).

${ }^{20}$ R. J. Tompkins, I. P. Mercer, M. Fettweis, C. J. Barnett, D. R. Klug, L. G. Porter, I. Clark, S. Jackson, P. Matousek, A. W. Parker, and M. Towrie,
Rev. Sci. Instrum. 69, 3113 (1998).

${ }^{21}$ M. Lamoureux, P. Waller, P. Charles, and N. B. Avdonina, Phys. Rev. E 62, 4091 (2000)

${ }^{22}$ M. J. Berger, J. S. Coursey, M. A. Zucker, and J. Chang, ESTAR: Electron Stopping-Power and Ranges, http://physics.nist.gov/PhysRefData/Star/ Text/ESTAR.html

${ }^{23} \mathrm{X}$-ray interactions with matter, http://www.cxro.lbl.gov/optical_constants/

${ }^{24}$ A. Thoss, Ph.D. Thesis, Fachbereich Physik der Freien Universität Berlin, 2003.

${ }^{25}$ F. Benesch, T. W. Lee, Y. Jiang, and C. G. Rose-Petruck, Opt. Lett. 29, 1028 (2004).

${ }^{26}$ A. Rousse, P. Audebert, J. P. Geindre, F. Fallies, J. C. Gauthier, A. Mysyrowicz, G. Grillon, and A. Antonetti, Phys. Rev. E 50, 2200 (1994).

${ }^{27}$ J. Steingruber, S. Borgstrom, T. Starczewski, and U. Litzen, J. Phys. B 29, L75 (1996).

${ }^{28}$ M. W. Bautz, G. Y. Prigozhin, M. J. Pivovaroff, S. E. Jones, S. E. Kissel, and G. R. Ricker, Nucl. Instrum. Methods Phys. Res. A 436, 40 (1999).

${ }^{29}$ G. Prigozhin, S. Jones, M. Bautz, G. Ricker, and S. Kraft, Nucl. Instrum. Methods Phys. Res. A 439, 582 (2000).

${ }^{30}$ P. Gibbon, Phys. Rev. Lett. 73, 664 (1994).

${ }^{31}$ X. Y. Peng, J. Zhang, T. J. Liang, Z. M. Sheng, Z. Jin, Y. T. Li, Z. H. Wang, Q. Z. Yu, Z. Y. Zheng, Y. Q. Liu, H. C. Wu, Z. Q. Hao, X. H. Yuan, and Z. Y. Wei, Chin. Phys. Lett. 21, 693 (2004).

${ }^{32}$ X. Y. Peng, J. Zhang, Z. Jin, T. J. Liang, J. Y. Zhong, H. C. Wu, Y. Q. Liu, Z. H. Wang, Z. L. Chen, Z. M. Sheng, Y. T. Li, and Z. Y. Wei, Acta Phys. Sin. 53, 2625 (2004).

${ }^{33}$ J. Zhang, Y. T. Li, Z. M. Sheng, Z. Y. Wei, Q. L. Dong, and X. Lu, Appl. Phys. B: Lasers Opt. 80, 957 (2005).

${ }^{34}$ A. Sjögren, M. Harbst, C. G. Wahlstrom, S. Svanberg, and C. Olsson, Rev. Sci. Instrum. 74, 2300 (2003).

${ }^{35}$ F. Brunel, Phys. Rev. Lett. 59, 52 (1987).

${ }^{36}$ K. Eidmann, R. Rix, T. Schlegel, and K. Witte, Europhys. Lett. 55, 334 (2001)

${ }^{37}$ C. N. Boyer, G. E. Holland, and J. F. Seely, Rev. Sci. Instrum. 76, 035109 (2005).

${ }^{38}$ L. Soto, Plasma Phys. Controlled Fusion 47, A361 (2005).

${ }^{39}$ Nuclear Instruments and Methods in Physics Research Section A: Accelerators, Spectrometers, Detectors and Associated Equipment (Elsevier, New York, 2006) Vol. 559, pp. 329-860.

${ }^{40}$ I. J. Maasilta, K. M. Kinnunen, A. K. Nuottajärvi, J. Leppäniemi, and A. Luukanen, Supercond. Sci. Technol. 19, S242 (2006).

${ }^{41}$ P. Beiersdorfer, R. E. Marrs, J. R. Henderson, D. A. Knapp, M. A. Levine, D. B. Platt, M. B. Schneider, D. A. Vogel, and K. L. Wong, Rev. Sci. Instrum. 61, 2338 (1990).

${ }^{42}$ A. P. Shevelko,Proc. SPIE 91, 3406 (1998).

${ }^{43}$ M. Kaburagi, Y. Z. Bin, D. Zhu, C. Y. Xu, and M. Matsuo, Carbon 41, 915 (2003).

${ }^{44}$ H. Legall, H. Stiel, V. Arkadiev, and A. A. Bjeoumikhov, Opt. Express 14, 4570 (2006).

${ }^{45}$ S. Bastiani, A. Rousse, J. P. Geindre, P. Audebert, C. Quoix, G. Hamoniaux, A. Antonetti, and J. C. Gauthier, Phys. Rev. E 56, 7179 (1997).

${ }^{46}$ P. P. Rajeev, P. Taneja, P. Ayyub, A. S. Sandhu, and G. R. Kumar, Phys. Rev. Lett. 90, 115002 (2003).

${ }^{47}$ M. Grätz, Ph.D. Thesis Lunds Universitet, 1998. 